\title{
Waist-to-Height Ratio, An Optimal Anthropometric Indicator in Prediction of Metabolic Dysfunction Associated Fatty Liver Disease: Results From A Chinese Male Population Survey
}

jinwei cai ( $\square$ caijinwei@gxmu.edu.cn )

Guangxi Medical University First Affiliated Hospital https://orcid.org/0000-0002-2688-9640

Cuiting Lin

Southern Medical University

Shuiqing Lai

Guangdong Provincial People's Hospital

Yingshan Liu

Southern Medical University

Min Liang

Guangxi Medical University First Affiliated Hospital

Yingfen Qin

Guangxi Medical University First Affiliated Hospital

Xinghuan Liang

Guangxi Medical University First Affiliated Hospital

Aihua Tan

Guangxi Medical University

Yong Gao

Guangxi Medical University

Zheng Lu

Guangxi Medical University

Chunlei Wu

Guangxi Medical University

Huang Shengzhu

Guangxi Medical University

Xiaobo Yang

Guangxi Medical University

Haiying Zhang

Guangxi Medical University

Jian Kuang

Guangdong Provincial People's Hospital

Zengnan Mo

Guangxi Medical University

\section{Research Article}

Keywords: Waist-to-height ratio, Lipid accumulation product, Abdominal volume index, Anthropometric indicator, Visceral fat, Metabolic-dysfunction associated fatty liver disease, Non-alcoholic fatty liver disease

Posted Date: August 3rd, 2021

DOI: https://doi.org/10.21203/rs.3.rs-762020/v1 
License: (9) (1) This work is licensed under a Creative Commons Attribution 4.0 International License. Read Full License 


\section{Abstract}

Background

Limited available evidence implicated that the anthropometric indicators of adiposity may contribute to the predictive and diagnosis capability of non-alcoholic fatty liver disease (NAFLD) in a facile, low-cost and noninvasive way while NAFLD had been entitled as metabolic-dysfunction associated fatty liver disease (MAFLD) in 2020. This study aimed to validate and compare the predictive and diagnostic capability of eight anthropometric indicators in MAFLD individuals and determined an optimal diagnostic predictor for MAFLD, which may beneficial for resource scarce regions.

Methods

The retrospective cross-sectional population-based study conducted from Fangchenggang Area Male Health and Examination Survey (FAMHES) involved 2428 participants whose comprehensive questionnaire, detailed data, anthropometric parameters and biochemical measurements were collected. Eight anthropometric indicators including body mass index (BMI), waist-to-height ratio (WHtR), waist-hip ratio (WHR), body adiposity index (BAl), cardiometabolic index (CMI), lipid accumulation product (LAP), visceral adiposity index (VAI) and Abdominal volume index( $\mathrm{AVI})$ were enrolled into analyzed. Receiver operating characteristic $(\mathrm{ROC})$ curve analysis and the area under the ROC curves (AUCs) were used to compare the diagnostic ability of each indicator for MAFLD and optimal cut-off points fully determined. Binary logistic regression analysis was used to explore associations of all anthropometric indicators and MAFLD by determined the odds ratios (ORs) and 95\% confidence interval (Cl).

\section{Results}

All selected anthropometric indicators were substantially associated with MAFLD $(p<0.001)$ and their diagnostic value of MAFLD by AUCs fully above 0.79 . WHtR [OR $22.181(95 \% \mathrm{Cl}, 16.216-30.340)$ ] had the strongest association with MAFLD, regardless of potential confounders. LAP had the largest AUC [0.868(95\% Cl 0.853-0.883)], intimately followed by WHtR [0.863(95\% $\mathrm{Cl} 0.848-0.879)]$. AVI with OR $19.275(95 \% \mathrm{Cl} 14.266-26.041)$ and AUC $0.859(95 \% \mathrm{Cl} 0.843-0.874)$ ranked second and third respectively among all indicators. The cut-off points of WHtR, LAP and AVI were $0.49,24.29$, and 13.61, separately.

\section{Conclusion}

All eight anthropometric indicators illustrated the superior diagnostic value of MAFLD. Comparatively, WHtR might stressed as the most powerful diagnostic predictor for MAFLD, follow by LAP and AVI which presented highly potential as well.

\section{Background}

By crude guestimates, NAFLD affects nearly a quarter of the global adult population or maybe more [1, 2]. The current definition of NAFLD reported in existing literature and guidelines is based on the presence of $>5 \%$ hepatocytes steatosis in liver and lack of significant sustained or recent alcohol consumption and other known causes of hepatic disease [1]. Habitually sedentary behavior, insufficient physical activity, uncontrolled high-calorie diet intake and imbalanced nutritional expenditure dramatically fueled the NAFLD onset and progression, these emerging unhealthy evidence response for NAFLD severity and mortality [3]. Especially noteworthy, epidemiological surveillance data unveiled that China had the highest prevalence, incidence, and annual NAFLD-related mortality rate throughout Asia [4]. Nevertheless, NAFLD until recently was not generally considered a serious public health issue in China [5].

Remarkably, NAFLD has been defined by a more constructive and novel criteria, entitled MAFLD in 2020. According to the current understanding of the booming prevalence and the pathogenesis of MAFLD, the new term had been grounded in a more comprehensive, overarching and appropriate way $[1,3]$. The novel suggested diagnostic criteria of MAFLD are built on the factual evidence of hepatic steatosis, chaperoning the coexistence of overweight/obesity or type 2 diabetes mellitus (T2DM); or the presence of hepatic steatosis with the coexistence of two other risk factors related to metabolic dysfunction in lean/normal weight individuals, regardless of alcohol consumption or other concomitant hepatic diseases $[1,6]$. A recent study claimed that MAFLD had better accuracy and practicability for identifying patients who suffered fatty liver at high risk of hepatic disease progression compared to NAFLD [7]. Therefore, MAFLD comprised a complete new set of diagnostic criteria, which is different from, but encompasses and beyond, NAFLD. 
Obesity substantially contributes to the risk of NAFLD [8]. Obesity and NAFLD have seen rising exponentially both in China and worldwide by parallel $[2,5,9]$. Mechanistically, hypertrophic obesity leads to dysfunctional and dysregulated subcutaneous adipose tissue and subsequently, the excess accumulation of ectopic fat in many depots including liver [10]. The accumulation of visceral fat has been considered the major risk factor as well as the most important predictor for NAFLD, being highly close related to the severity of the disease, particularly in the setting of dietary overindulgence $[11,12]$.

Recent researches demonstrated that the content and accumulation of adipose tissue can be evaluated using various anthropometric indicators which used to be markers of visceral fat distribution and dysfunction [13-15]: Waist circumference (WC), BMI [16], LAP[17], WHR[18],WHtR [18], VAI[9, 19], and BAI[20]. Currently, the most sensitive and specific indicators for discriminating visceral fat have been described in the literature as WHtR and LAP, compared to the classic parameters as WC and BMI [21]. Meanwhile, WHR, WHtR and LAP had been regarded as good predictors of NAFLD $[11,12]$. Comparatively, body roundness index (BRI) [22] had been requested to be cautiously applied in the assessment of diagnostic capability of NAFLD, and body shape index (ABSI)[23] was identified as a poor predictor for NAFLD simultaneously [12]. Limited available evidence implicated that the anthropometric indicators of adiposity may contribute to the predictive and diagnosis capability of NAFLD in a facile, low-cost and noninvasive way despite the controversy regarding the most appropriate anthropometric indicator to predict NAFLD is yet to be elucidated [11].

Additionally, recent evidence explored that the racial/ethnic prevalence of obesity did not completely parallel NAFLD risk, incremental weight gain in Asians appeared more detrimental [24]. NAFLD has significant racial/ethnic variability both in the prevalence and characteristics. Of note, the fact that Chinese people have substantially higher risks of NAFLD while at much lower BMI levels compared with the Unite states population, which indicates a strong ethnic heterogeneity in anthropometric indicators and its association with NAFLD [5]. Meanwhile, a meta-analysis revealed that the Asian populations (OR:3.74 [95\% $\mathrm{Cl}, 2.51-5.55]$ ) have higher NAFLD risk associated with obesity or increased BMI compared to Caucasian populations (OR: 2.67 [95\% Cl, 1.58-4.52]) [25]. Hitherto, a large amount of NAFLD patients remain undiagnosed because of the inadequacy of diagnostic tools for convenient, cost-effective clinical assessment and routine screen $[4,5]$. Thus, it is imperative to validate an effective tool for predict and diagnosis MAFLD while balancing both generalizability and eligibility.

To address this gap, herein we collected eight anthropometric indicators (BMI, WHtR, WHR, BAI, LAP, VAI, CMI [26] and AVI [27]) which reflecting visceral fat and are highly conducive to calculate, aimed to assess predictive capability as well as the optimal cut-off points of these novel indicators in individuals with MAFLD. Meanwhile, we hypothesized that anthropometric indicators of adiposity have strongly racial/ethnic disparities in reflecting liver fat deposition. Moreover, since much of the literature on NAFLD prevalence and anthropometric indicators of adiposity were mainly conducted with white ancestry in the developed areas as the reference group, which constrains generalizability to other populations and less developed regions. Thus, it is essential to further validate the predictive and diagnostic capacity of selected eight anthropometric indicators in MAFLD individuals by a large-scale Chinese male population and determine the accuracy of candidate indicators for optimal prediction and discrimination of MAFLD in Western China.

\section{Materials And Methods}

\section{Study design and patient selection}

The current research was performed using a survey from a cross-sectional population-based study, FAMHES, which was completely described previously [28-31]. Briefly, FAMHES was conducted in Fangchenggang city which located in Guangxi Zhuang Autonomous Region of Western China, namely underdeveloped regions. The study was designed to reveal the effects of environmental and genetic factors in non-hospitalized Chinese male individuals involving their interaction with the development of age-related chronic diseases. Local men aged $\geq 18$ years were convened to participate in the survey upon large-scale health examination at the Medical Center of Fangchenggang First People's Hospital from September 2009 to December 2009. Eventually, a total of 4303 men aged between 18 to 88 year were recruited with their comprehensive demographic and physical examination data exhaustively collected. After exclusions (self-reported cancer, currently diagnosed with chronic disease, acute infectious diseases, use any drug that might affect the endocrine system), 2428 participants were enrolled. Furthermore, after excluded subjects with incomplete or irrational data involved anthropometric and ultrasonography, 2355 men were qualified for the study, and 515 were diagnosed as MAFLD by the novel expert consensus statement announced in 2020(Fig. 1) [1]. Written informed consent was obtained from all participants. The study received approval from the Medical Ethics Committee of First Affiliated Hospital of Guangxi Medical University.

\section{Data collection}


A detailed and standardized structural questionnaire was conducted by trained physicians for all participants. The comprehensive collected data including demographic information (age, education, occupation, financial status, etc.), lifestyle characteristics (smoking and alcohol consumption history, physical activity), health status (self-reported medical history and current medications) and family history of chronic diseases. Anthropometric parameters were undergone by trained personnel using a standardized protocol. Body shape measurements including waist, hip, and thigh circumferences which were acquired while the participants were dressed in thin clothes without shoes. Weight and height were measured by digital scales to the nearest $0.1 \mathrm{~kg}$ and $0.1 \mathrm{~cm}$ accuracy, respectively. The waist and hip circumferences were measured by a non-stretching tape to the nearest $0.1 \mathrm{~cm}$. WC was determined to approximate the midpoint between the lowest margin of the last palpable rib and the top of the iliac. Hip circumference $(\mathrm{HC})$ was measured around the widest portion of the buttocks axial plane [28, 32,33]. Blood pressure was measured as the mean of the first 2 measurements taken in a seated position using the right arm by a mercury sphygmomanometer, or an automated device after at least 5-minute rest. Participants were required to avoid vigorous exercise, drinking, and smoking for at least 30 minutes before the measurement [28].

\section{Biochemical measurements}

Blood samples were drawn from the ulnar vein between 7 am to 9 am after overnight fasting, and used to assay triglyceride (TG), total cholesterol (TC), high-density lipoprotein (HDL), low-density lipoprotein (LDL), serum alanine aminotransferase (ALT), uric acid(UA), fasting serum insulin, fasting plasma glucose (FPG). Lipid parameters, fasting serum insulin, UA and FPG were measured enzymatically on Dimension-RxL Chemistry Analyzer (Dade Behring, Newark, DE) in the Department of Clinical Laboratory at the Fangchenggang First People's Hospital [28, 29]. Meanwhile, follicle stimulating hormone(FSH), luteinizing hormone (LH), total testosterone (TT), Estradiol(E2) and serum sex hormone-binding globulin (SHBG) were measured with electrochemiluminescence immunoassay on COBAS 6000 system E601 (Elecsysmodule) immunoassay analyzers (Roche Diagnostics, IN, Germany). The interassay coefficient of variation was $4.3 \%$ for $\mathrm{FSH}, 3.6 \%$ for $\mathrm{LH}, 3.4 \%$ for $\mathrm{E} 2,3.6 \%$ for $\mathrm{T}$ and $4.4 \%$ for SHBG. All assays were conducted according to the standard manufacturer's instructions as previously described $[29,31,34]$.

\section{Candidate anthropometric predictors}

All selected candidate anthropometric indicators were calculated by following standard formulas:

$$
\begin{aligned}
& \text { BMI = Weight }(\mathrm{kg}) / \text { Height }(\mathrm{m})^{2}\left(\mathrm{~kg} / \mathrm{m}^{2}\right) \text { [16] } \\
& \text { WHtR }=\text { WC }(\mathrm{cm}) / \text { Height }(\mathrm{cm})[18] \\
& \text { WHR = WC }(\mathrm{cm}) / \mathrm{HC}(\mathrm{cm})[18] \\
& \mathrm{BAI}=\left[\mathrm{HC}(\mathrm{cm}) / \text { Height }(\mathrm{m})^{1.5}\right]-18[20] \\
& \mathrm{CMI}=\mathrm{WH} \mathrm{tR} \times \mathrm{TG} / \mathrm{HDL}[26] \\
& \text { LAP }=[\mathrm{WC}(\mathrm{cm})-65] \times T \mathrm{TG}(\mathrm{mmol} / \mathrm{L}) \text { in Male [17] } \\
& \mathrm{VAl}=\mathrm{WC}(\mathrm{cm}) /[39.68+(1.88 \times \mathrm{BMI})] \times[(\mathrm{TG} / 1.03) \times(1.31 / \mathrm{HDL})] \text { in male }[19] \\
& \mathrm{AVI}=\left[2 \times \mathrm{cm}(\mathrm{WC})^{2}+0.7 \times \mathrm{cm}(\mathrm{WC}-\mathrm{HC})^{2}\right] / 1000[27] \\
& \text { Insulin resistance indices }
\end{aligned}
$$

Insulin resistance (IR), were calculated by the standard formulas:

HOMA-IR = fasting Insulin $(\mu \mathrm{IU} / \mathrm{mL}) \times F P G(\mathrm{mmol} / \mathrm{L}) / 22.5$

HOMA- $\beta=20 \times$ fasting Insulin ( $\mu \mathrm{lU} / \mathrm{mL}) /[\mathrm{FPG}(\mathrm{mmol} / \mathrm{L})-3.5][35]$

Or HOMA-IR= [FPG $(\mathrm{mg} / \mathrm{dL}) \times$ fasting Insulin $(\mu \mathrm{IU} / \mathrm{mL})] / 405$

HOMA- $\beta=360 \times$ fasting Insulin $(\mu \mathrm{lU} / \mathrm{mL}) /[\mathrm{FPG}(\mathrm{mg} / \mathrm{dL})-63]$ [36]

HOMA\% Sensitivity (HOMA-IS) was calculated as reciprocal of HOMA-IR (1/HOMA-IR) [35]

\section{Ultrasonography}


The abdominal ultrasonography for all participants was performed using a portable ultrasound device (GE, LOGIQ e, 5.0MHz transducer, Fairfield, CT, USA) by two experienced ultrasonographers. All individuals were assessed for liver status including size, echogenicity, contour, structure and posterior beam attenuation. The ultrasound diagnostic criteria of fatter liver were based on the following items: diffused liver enhanced near field echo, with stronger echoes in the hepatic parenchyma than that of the kidney; dirty liver far field echo decays; intrahepatic duct structure display blurring [31, 34, 37].

\section{Definition of MAFLD}

The novel positive diagnostic criteria of MAFLD was particularly noteworthy in the following content: (1) Regardless of alcohol consumption or other concomitant liver diseases; (2) Based on hepatic histological (biopsy), imaging or blood biomarker evidence of fat accumulation in the liver (hepatic steatosis); (3) In addition to one of the major three situations, namely overweight/obesity, presence of T2DM, or evidence of metabolic dysregulation; (4) The latter must defined by the presence of at least two metabolic risk abnormalities which have been described in more detail[1].

\section{Statistical analysis}

The demographic and clinical data of the study subjects were presented using mean \pm standard deviation for normality distribution and median (interquartile range) for skewed distribution as continuous variables. Comparisons of Clinical characteristics and adipose tissue accumulation indicators between groups (MAFLD and non-MAFLD) were made using Students $t$-test and Mann-Whitney rank sum tests appropriately. The normal percentile method was adopted to formulate the estimated reference range for all adipose tissue accumulation indicators, which were represented by P2.5-P97.5. ROC curve analysis was used to compare the diagnostic ability of each indicator for MAFLD according to the AUC. The indicator with the largest AUC was accepted as the most valuable indicator. The optimal cut-off value was determined with the maximal "Youden index" (defined as [Sensitivity + Specificity-1]) [38]. Binary logistic regression models were used to explore associations between anthropometric indicators and MAFLD. Potential confounding variables including age (continuous), blood glucose, blood pressure, plasma uric acid, lipid parameters and sex hormone parameters were entered into models in a stepwise manner. Statistical analysis was conducted with SPSS version 25.0(IBM). Statistical tests were performed as 2-sided, and $P<0.05$ was assumed statistically significant.

\section{Results}

\section{Subject Characteristic}

The total 2355 enrolled participants were divided into MAFLD group $(n=515)$ and non-MAFLD group $(n=1840)$ according to the novel consensus of diagnostic criteria [1]. The prevalence of MAFLD among all participants was $21.87 \%$. Compared to the individuals without MAFLD, age, weight, WC, HC, systolic blood pressure (SBP), diastolic blood pressure (DBP), ALT, TC, TG, LDL, UA, FPG, Insulin, HOMA-IR and HOMA- $\beta$ were significantly higher ( $p<0.001$;Table 1), while HDL, HOMA-IS, LH, E2, TT and SHBG were substantially lower among individuals with MAFLD ( $p<0.001 ;$ Table 1). Meanwhile, subject height and FSH concentration present no difference between the two groups (Table 1). 
Table 1

Baseline characteristics in study male population.

\begin{tabular}{|c|c|c|c|c|c|}
\hline Characteristics & $\begin{array}{l}\text { Total } \\
(n=2355)\end{array}$ & $\begin{array}{l}\text { MAFLD } \\
(n=515)\end{array}$ & $\begin{array}{l}\text { Non-MAFLD } \\
(n=1840)\end{array}$ & $\begin{array}{l}t \text {-Value } \\
Z \text {-Value }\end{array}$ & $P$-Value \\
\hline Age(years) & $37.82 \pm 11.10$ & $39.84 \pm 9.92$ & $37.26 \pm 11.35$ & 21.90 & $<0.001$ \\
\hline Height (cm) & $167.97 \pm 5.54$ & $167.98 \pm 5.61$ & $167.97 \pm 5.53$ & 0.002 & 0.967 \\
\hline Weight (kg) & $65.87 \pm 10.43$ & $74.85 \pm 9.58$ & $63.36 \pm 9.21$ & 614.606 & $<0.001$ \\
\hline $\mathrm{WC}(\mathrm{cm})$ & $80.87 \pm 9.23$ & $89.90 \pm 6.75$ & $78.34 \pm 8.19$ & 861.693 & $<0.001$ \\
\hline $\mathrm{HC}(\mathrm{cm})$ & $91.51 \pm 6.20$ & $96.56 \pm 5.59$ & $90.10 \pm 5.60$ & 535.883 & $<0.001$ \\
\hline $\mathrm{SBP}(\mathrm{mmHg})$ & $118.32 \pm 15.48$ & $123.53 \pm 17.00$ & $116.87 \pm 14.70$ & 77.045 & $<0.001$ \\
\hline $\mathrm{DBP}(\mathrm{mmHg})$ & $77.00 \pm 10.25$ & $81.23 \pm 11.34$ & $75.82 \pm 9.60$ & 117.440 & $<0.001$ \\
\hline $\operatorname{ALT}(\mathrm{U} / \mathrm{I})$ & $39.00(30.00-54.00)$ & $54.00(40.00-74.00)$ & $36.00(29.00-48.00)$ & -16.349 & $<0.001$ \\
\hline \multicolumn{6}{|l|}{ Lipid profile } \\
\hline $\mathrm{TC}(\mathrm{mmol} / \mathrm{l})$ & $5.71 \pm 1.03$ & $6.08 \pm 1.03$ & $5.60 \pm 1.00$ & 91.722 & $<0.001$ \\
\hline TG (mmol/l) & $1.13(0.78-1.75)$ & $1.88(1.31-2.79)$ & $1.00(0.71-1.48)$ & -20.072 & $<0.001$ \\
\hline $\mathrm{HDL}(\mathrm{mmol} / \mathrm{l})$ & $1.40 \pm 0.33$ & $1.29 \pm 0.40$ & $1.44 \pm 0.30$ & 77.731 & $<0.001$ \\
\hline LDL (mmol/l) & $2.97 \pm 0.80$ & $3.28 \pm 0.78$ & $2.88 \pm 0.79$ & 103.852 & $<0.001$ \\
\hline \multicolumn{6}{|l|}{ Metabolic indices } \\
\hline $\mathrm{UA}(\mu \mathrm{mol} / \mathrm{l})$ & $378.46 \pm 79.681$ & $420.29 \pm 8704$ & $366.76 \pm 73.37$ & 196.666 & $<0.001$ \\
\hline FPG $(\mathrm{mmol} / \mathrm{l})$ & $5.20(4.90-5.60)$ & $5.40(5.00-5.80)$ & $5.10(4.90-5.50)$ & -7.989 & $<0.001$ \\
\hline Insulin(mU/I) & $6.38(4.27-9.67)$ & $10.46(7.57-15.22)$ & $5.61(3.85-8.19)$ & -20.597 & $<0.001$ \\
\hline HOMA-IR & $1.47(0.97-2.31)$ & $2.55(1.80-3.75)$ & $1.29(0.87-1.90)$ & -21.091 & $<0.001$ \\
\hline HOMA-IS & $0.68(0.43-1.03)$ & $0.39(0.27-0.56)$ & $0.78(0.53-1.14)$ & -21.091 & $<0.001$ \\
\hline HOMA- $\beta(\%)$ & 78.35(49.82-118.33) & 115.08(79.45-164.45) & 69.82(45.86-104.74) & -14.955 & $<0.001$ \\
\hline \multicolumn{6}{|l|}{ Hormones } \\
\hline $\mathrm{FSH}(\mathrm{mlU} / \mathrm{ml})$ & $5.10(3.58-7.53)$ & $5.06(3.63-7.53)$ & $5.12(3.56-7.54)$ & -0.248 & 0.804 \\
\hline $\mathrm{LH}(\mathrm{mlU} / \mathrm{ml})$ & $5.18(3.97-6.72)$ & $4.89(3.67-6.42)$ & $5.27(4.05-6.81)$ & -4.053 & $<0.001$ \\
\hline Estradiol (pg/ml) & $34.34 \pm 10.13$ & $32.58 \pm 8.73$ & $34.84 \pm 10.44$ & 20.075 & $<0.001$ \\
\hline Testosterone (ng/ml) & $6.26 \pm 1.93$ & $4.92 \pm 1.48$ & $6.64 \pm 1.87$ & 365.606 & $<0.001$ \\
\hline SHBG(nmol/l) & $38.35(28.02-51.28)$ & $25.84(19.43-35.04)$ & 41.97(32.10-54.44) & -19.811 & $<0.001$ \\
\hline \multicolumn{6}{|c|}{$\begin{array}{l}\text { Abbreviations: MAFLD: Metabolic-dysfunction associated fatty liver disease; WC. Waist circumference; HC. HIP circumference; } S B P \text { : } \\
\text { Systolic blood pressure; } D B P \text { : Diastolic blood pressure; } A L T \text { : Alanine transaminase; TC. Total cholesterol; TG: Triglyceride; HDL: } \\
\text { High-density lipoprotein; } L D L \text { : Low-density lipoprotein; UA: Uric acid; FPG: Fasting plasma glucose; HOMA: Homeostatic model } \\
\text { assessment; FSH: Follicle stimulating hormone; } L H \text { : Luteinizing hormone; } S H B G \text { : Sex hormone-binding globulin. Numeric variables } \\
\text { are described by (mean } \pm S D \text { ) or median (interquartile range). } P<0.05 \text { was accepted statistically significant. }\end{array}$} \\
\hline
\end{tabular}

The Mean \pm Standard Deviation and recommended reference range of anthropometric indicators between individuals with and without MAFLD for men

Table 2 showed the mean \pm standard deviation for the anthropometric indicators in individuals with and without MAFLD for men. According to our results, the dramatically higher values of anthropometric indicators were related to MAFLD sufferers as compared to others $(p<0.001$; Table 2$)$. We established recommended reference range of anthropometric indicators by the 2.5 th and 97.5 th 
percentiles (Table 3). The reference of each selected anthropometric indicator in subjects with MAFLD is significantly higher than those without MAFLD.

Table 2

Mean \pm standard deviation and median (interquartile range) for the anthropometric indicators in individuals. ( $n=2355$ )

\begin{tabular}{|c|c|c|c|c|c|}
\hline & $\begin{array}{l}\text { Total } \\
(n=2355)\end{array}$ & $\begin{array}{l}\text { MAFLD } \\
(n=515)\end{array}$ & $\begin{array}{l}\text { Non-MAFLD } \\
(n=1840)\end{array}$ & $\begin{array}{l}t-\text { Value } \\
Z \text {-Value }\end{array}$ & $P$-Value \\
\hline BMI & $23.32 \pm 3.35$ & $26.49 \pm 2.82$ & $22.44 \pm 2.92$ & 786.688 & $<0.001$ \\
\hline WHtR & $0.48 \pm 0.05$ & $0.54 \pm 0.04$ & $0.46 \pm 0.05$ & 887.109 & $<0.001$ \\
\hline WHR & $0.88 \pm 0.06$ & $0.93 \pm 0.05$ & $0.87 \pm 0.05$ & 583.939 & $<0.001$ \\
\hline $\mathrm{BAl}$ & $24.08 \pm 2.94$ & $26.39 \pm 2.65$ & $23.43 \pm 2.68$ & 493.532 & $<0.001$ \\
\hline CMI & $0.39(0.24-0.72)$ & $0.84(0.54-1.28)$ & $0.32(0.21-0.55)$ & -22.183 & $<0.001$ \\
\hline LAP & $17.09(7.02-37.00)$ & $45.24(30.97-71.40)$ & $12.09(5.40-24.61)$ & -25.596 & $<0.001$ \\
\hline VAl & $0.81(0.50-1.43)$ & $1.64(1.02-2.60)$ & $0.68(0.45-1.13)$ & -21.308 & $<0.001$ \\
\hline AVI & $13.35 \pm 2.99$ & $16.30 \pm 2.45$ & $12.52 \pm 2.58$ & 881.957 & $<0.001$ \\
\hline \multicolumn{6}{|c|}{$\begin{array}{l}\text { Abbreviations: MAFLD: Metabolic-dysfunction associated fatty liver disease; } B M I \text { : Body mass index; WHtR: Waist to height ratio; } \\
\text { WHR: Waist to hip ratio; } B A l \text { : Body adiposity index; } C M \text { : Cardiometabolic index; } L A P \text {. Lipid accumulation product; VAl: Visceral } \\
\text { adiposity index; } A V \text { : Abdominal volume index. Numeric variables are described by (mean } \pm S D \text { ) or median (interquartile range). } P \\
0.05 \text { was accepted statistically significant. }\end{array}$} \\
\hline
\end{tabular}

Table 3

Recommended reference range of the anthropometric indicators in individuals. $(n=2355)$

\begin{tabular}{|c|c|c|c|}
\hline & $\begin{array}{l}\text { Total } \\
(n=2355)\end{array}$ & $\begin{array}{l}\text { MAFLD } \\
(n=515)\end{array}$ & $\begin{array}{l}\text { Non-MAFLD } \\
(n=1840)\end{array}$ \\
\hline BMI & $17.75-30.63$ & $21.03-32.91$ & $17.61-28.68$ \\
\hline WHtR & $0.39-0.59$ & $0.46-0.62$ & $0.39-0.56$ \\
\hline WHR & 0.77-1.00 & $0.85-1.03$ & $0.77-0.98$ \\
\hline BAl & $18.70-30.33$ & $21.77-31.98$ & $18.40-28.99$ \\
\hline CMI & $0.10-2.36$ & $0.27-3.23$ & $0.10-1.74$ \\
\hline LAP & $0.31-122.29$ & $12.53-192.47$ & $0.00-77.54$ \\
\hline VAl & $0.23-4.80$ & $0.53-6.50$ & $0.21-3.54$ \\
\hline AVI & $8.83-19.72$ & $12.11-21.78$ & $8.68-18.09$ \\
\hline \multicolumn{4}{|c|}{$\begin{array}{l}\text { Abbreviations: MAFLD: Metabolic-dysfunction associated fatty liver disease; } B M \text { : Body mass index; WHtR: Waist to height ratio; } \\
\text { WHR: Waist to hip ratio; } B A \text { : Body adiposity index; } C M \text { : Cardiometabolic index; } L A P \text { : Lipid accumulation product; } V A \text { : Visceral } \\
\text { adiposity index; } A V I \text { : Abdominal volume index. The recommended reference range of anthropometric indicators were defined by the } \\
\text { 2.5th and } 97.5 \text { th percentiles. }\end{array}$} \\
\hline
\end{tabular}

The cut-off points and area under the receiver operating characteristic curves ( $95 \%$ confidence interval)of anthropometric indicators and HOMA-index in predicting MAFLD.

We assessed the diagnostic abilities of selected indicators in the diagnosis of MAFLD.ROC curves were analyzed by setting optimal cut-off points for each indicator. The AUC analysis results revealed that all eight selected anthropometric indicators had diagnostic value for MAFLD and LAP(AUC:0.868 [95\% Cl,0.853-0.883])had been confirmed with the largest AUC which reflecting the highest diagnostic value, followed by WHtR(0.863 [0.848-0.879]), AVI(0.859 [0.843-0.874]), BMI(0.846 [0.829-0.864]), CMI(0.819 [0.800$0.838]), \operatorname{WHR}(0.814[0.796-0.833]), \operatorname{VAl}(0.807$ [0.787-0.826]), and $\mathrm{BAI}(0.790[0.769-0.810])$. The optimal cut-off points at which the risk of MAFLD increased was 24.0 (sens: sensitivity: $84.08 \%$, spec: specificity: $72.39 \%$ ) for BMI, 0.49 (sens: $90.68 \%$, spec: 
69.56\%) for WHtR, 0.89(sens:84.46\%,spec:64.95\%) for WHR, 24.97(sens:71.7\%,spec:72.50\%) for BAl, 0.48(sens:81.75\%,spec:69.24\%) for CMI, 24.29(sens:86.60\%,spec:74.73\%) for LAP, 0.94(sens:81.20\%,

spec:67.23\%) for VAl, 13.61(sens:89.71\%,spec:68.86\%) for AVI, 1.69 (sens:81.36\%,spec:68.91\%) for HOMA-IR, 78.1(sens:76.31\%,spec:57.18

\%) for HOMA- $\beta$ (Table 4; Fig. 2). When cut-off point determined, WHtR was noticed had the highest sensitivity (90.68\%), whereas LAP had the best specificity $(74.73 \%)$, separately.

Table 4

Cut-off points and AUCs $(95 \% \mathrm{Cl})$ of different anthropometric indicators and HOMA-index in predicting MAFLD $(\mathrm{n}=2355)$

\begin{tabular}{|c|c|c|c|c|}
\hline & $\operatorname{AUC}(95 \% \mathrm{Cl})$ & Cut-off piont & Sensitivity (\%) & Specificity (\%) \\
\hline BMI & $0.846(0.829-0.864)$ & 24.00 & 84.08 & 72.39 \\
\hline WHtR & $0.863(0.848-0.879)$ & 0.49 & 90.68 & 69.56 \\
\hline WHR & $0.814(0.796-0.833)$ & 0.89 & 84.46 & 64.95 \\
\hline $\mathrm{BAI}$ & $0.790(0.769-0.810)$ & 24.97 & 71.70 & 72.50 \\
\hline $\mathrm{CMI}$ & $0.819(0.800-0.838)$ & 0.48 & 81.75 & 69.24 \\
\hline LAP & $0.868(0.853-0.883)$ & 24.29 & 86.60 & 74.73 \\
\hline VAI & $0.807(0.787-0.826)$ & 0.94 & 81.20 & 67.23 \\
\hline AVI & $0.859(0.843-0.874)$ & 13.61 & 89.71 & 68.86 \\
\hline HOMA-IR & $0.803(0.783-0.824)$ & 1.69 & 81.36 & 68.91 \\
\hline HOMA- $\beta$ & $0.715(0.690-0.740)$ & 78.10 & 76.31 & 57.18 \\
\hline
\end{tabular}

\section{Binary logistic regression analysis of anthropometric indicators in predicting MAFLD}

The statistical results explored that all anthropometric indicators were substantially associated with MAFLD $(p<0.001 ;$ Table 5$)$. WHtR [OR 22.181(95\% Cl,16.216-30.340)] had been observed to have the strongest association with MAFLD which indicated the most effective prediction capability for MAFLD, followed by AVI, LAP, BMI, CMI, WHR, HOMA-IR, VAI, BAI and HOMA- $\beta$. After adjustment for potential confounding variables including age (continuous), blood glucose, blood pressure, plasma uric acid, lipid parameters and sex hormone parameters separately (model I, II and III), ORs of selected indicators decreased dramatically in all models. Interestingly, WHtR still is the indicator with the highest OR value, which indicates the strongest prediction capability for MAFLD regardless underwent any controlled models (Table 5). The results of the multivariate binary logistic regression models indicated that with the adjustment of the models, advanced age, hypertension, hyperuricemia, hypertriglyceridemia and FSH were risk factors for MAFLD, while LH, TT, SHBG

were the protective factors (Supplementary Table S1). The results were generally consistent with the findings of previous studies [8,31, $37,39,40]$. In model II, HDL was a protective factor and diabetes was a risk factor, but after adjusting for sex hormones and SHBG (model III), HDL was no more a statistically significant protective factor for MAFLD in CMI, LAP and VAl models, and hypertriglyceridemia was no longer a statistically significant risk factor in CMI and LAP models. Plasma glucose also was no longer a significant factor for MAFLD in all anthropometric models except BAl. Details of multivariate binary logistic regression analysis are presented in Supplementary Table $\mathbf{S 1 .}$ 
Table 5

Binary logistic regression analysis of anthropometric indicators and HOMA-index in predicting MAFLD ( $n=2355)$

\begin{tabular}{|c|c|c|c|c|c|c|c|c|}
\hline \multirow[t]{2}{*}{ Variable } & \multicolumn{2}{|l|}{ Non-adjusted } & \multicolumn{2}{|l|}{ Model I } & \multicolumn{2}{|l|}{ Model II } & \multicolumn{2}{|l|}{ Model III } \\
\hline & $\mathrm{OR}(95 \% \mathrm{Cl})$ & $\begin{array}{l}p \\
\text { value }\end{array}$ & $\mathrm{OR}(95 \% \mathrm{Cl})$ & $\begin{array}{l}p- \\
\text { value }\end{array}$ & $\mathrm{OR}(95 \% \mathrm{Cl})$ & $\begin{array}{l}p- \\
\text { value }\end{array}$ & $\mathrm{OR}(95 \% \mathrm{Cl})$ & $\begin{array}{l}p \\
\text { value }\end{array}$ \\
\hline BMI & $\begin{array}{l}13.722(10.620- \\
17.729)\end{array}$ & $<.001$ & $\begin{array}{l}12.768(9.866- \\
16.525)\end{array}$ & $<0.001$ & $\begin{array}{l}7.431(5.635- \\
9.800)\end{array}$ & $\begin{array}{l}< \\
0.001\end{array}$ & $\begin{array}{l}5.019(3.751- \\
6.715)\end{array}$ & <. \\
\hline WHtR & $\begin{array}{l}22.181(16.216- \\
30.340)\end{array}$ & $<001$ & $\begin{array}{l}21.441(15.575- \\
29.516)\end{array}$ & $\begin{array}{l}< \\
0.001\end{array}$ & $\begin{array}{l}12.454(8.880- \\
17.466)\end{array}$ & $\begin{array}{l}< \\
0.001\end{array}$ & $\begin{array}{l}7.795(5.492- \\
11.064)\end{array}$ & $<0.001$ \\
\hline WHR & $\begin{array}{l}10.050(7.773- \\
12.994)\end{array}$ & $<.001$ & $\begin{array}{l}9.583(7.335- \\
12.521)\end{array}$ & $\begin{array}{l}< \\
0.001\end{array}$ & $\begin{array}{l}5.468(4.107- \\
7.280)\end{array}$ & $\begin{array}{l}< \\
0.001\end{array}$ & $\begin{array}{l}3.924(2.912- \\
5.287)\end{array}$ & <. \\
\hline BAI & $\begin{array}{l}6.663(5.362- \\
8.280)\end{array}$ & $<.001$ & $\begin{array}{l}6.248(5.013- \\
7.786)\end{array}$ & $<0.001$ & $\begin{array}{l}4.014(3.159- \\
5.099)\end{array}$ & $<0.001$ & $\begin{array}{l}2.922(2.267- \\
3.767)\end{array}$ & <. \\
\hline CMI & $\begin{array}{l}10.081(7.894- \\
12.874)\end{array}$ & $<.001$ & $\begin{array}{l}9.459(7.394- \\
12.101)\end{array}$ & $\begin{array}{l}< \\
0.001\end{array}$ & $\begin{array}{l}4.969(3.589- \\
6.880)\end{array}$ & $<0.001$ & $\begin{array}{l}3.288(2.339- \\
4.621)\end{array}$ & $<.001$ \\
\hline LAP & $\begin{array}{l}16.856(12.825- \\
22.155)\end{array}$ & $<.001$ & $\begin{array}{l}15.734(11.952- \\
20.714)\end{array}$ & $<.001$ & $\begin{array}{l}9.029(6.560- \\
12.426)\end{array}$ & $\dot{0} 0.001$ & $\begin{array}{l}6.077(4.345- \\
8.501)\end{array}$ & $<.001$ \\
\hline VAl & $\begin{array}{l}8.818(6.927- \\
11.226)\end{array}$ & $<.001$ & $\begin{array}{l}8.331(6.532- \\
10.627)\end{array}$ & $<0.001$ & $\begin{array}{l}3.933(2.855- \\
5.417)\end{array}$ & $<0.001$ & $\begin{array}{l}2.685(1.917- \\
3.759)\end{array}$ & ¿ 0.001 \\
\hline AVI & $\begin{array}{l}19.275(14.266- \\
26.041)\end{array}$ & $<.001$ & $\begin{array}{l}18.107(13.352- \\
24.555)\end{array}$ & $\begin{array}{l}< \\
0.001\end{array}$ & $\begin{array}{l}10.359(7.498- \\
14.311)\end{array}$ & $\begin{array}{l}< \\
0.001\end{array}$ & $\begin{array}{l}6.537(4.676- \\
9.138)\end{array}$ & $\begin{array}{l}< \\
0.001\end{array}$ \\
\hline $\begin{array}{l}\text { HOMA- } \\
\text { IR }\end{array}$ & $\begin{array}{l}9.675(7.590- \\
12.334)\end{array}$ & $<.001$ & $\begin{array}{l}9.761(7.634- \\
12.481)\end{array}$ & $<.001$ & $\begin{array}{l}6.149(4.689- \\
8.064)\end{array}$ & $\begin{array}{l}<.001 \\
0.00\end{array}$ & $\begin{array}{l}4.384(3.298- \\
5.828)\end{array}$ & $<.001$ \\
\hline $\begin{array}{l}\text { HOMA- } \\
\beta\end{array}$ & $\begin{array}{l}4.281(3.425- \\
5.352)\end{array}$ & $<.001$ & $\begin{array}{l}4.710(3.748- \\
5.918)\end{array}$ & $<0.001$ & $\begin{array}{l}3.594(2.768- \\
4.666)\end{array}$ & $<0.001$ & $\begin{array}{l}2.792(2.110- \\
3.694)\end{array}$ & <. \\
\hline \multicolumn{9}{|c|}{$\begin{array}{l}\text { Abbreviations: MAFLD: Metabolic-dysfunction associated fatty liver disease; } B M l \text { : Body mass index; WHtR: Waist to height ratio; } \\
\text { WHR: Waist to hip ratio; } B A I \text { : Body adiposity index; } C M I \text { : Cardiometabolic index; } L A P \text {. Lipid accumulation product; VAl: Visceral } \\
\text { adiposity index; } A V l \text { : Abdominal volume index; HOMA: Homeostatic model assessment; OR: Odds ratio; } C l \text { : Confidence interval. } P< \\
0.05 \text { was accepted statistically significant. }\end{array}$} \\
\hline \multicolumn{9}{|c|}{ Model I: Adjusted for age } \\
\hline \multicolumn{9}{|c|}{ 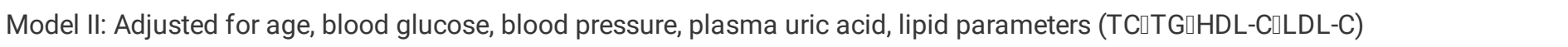 } \\
\hline
\end{tabular}

\section{Discussion}

The total NAFLD population has been estimated to increase to 314.58 million cases in China by 2030, the greatest increase in NAFLD prevalence globally [4]. Given the rapid geographic expansion and abrupt increase in the NAFLD population of China, tremendous economic and clinical burden is inevitable. The development of economical tools for widespread screening, prediction and diagnosis of MAFLD in the general population deserves further concern.

The prevalence of MAFLD in FAMHES conducted in Guangxi area turned out to be $21.87 \%$, significantly lower than the overall NAFLD prevalence of China nationwide over the past two decades[29.6\%(95\% Cl,28.2\%-31.0\%)][4]. Furthermore, the prevalence of NAFLD throughout the country had reached $32.9 \%(95 \% \mathrm{Cl}, 28.9 \%-36.8 \%)$ in $2018[4]$. The counterintuitive outcome reflecting complex yet potentially modifiable interaction between NAFLD prevalence and the area urbanization along with industrialization. According to the latest meta-analysis, the national gross domestic product (GDP) rank was inversely associated with the prevalence of NAFLD in the past two decades in China. Meanwhile, the emerging available studies regard to the prevalence of NAFLD were mainly within the region with higher GDP per capita in recent decades. The few studies performed in less developed regions may attribute to larger bias and should warrant more attention [5]. Clinical vigilance for MAFLD should be maintained without exclusion in less developed areas. 
In the present study, eight selected anthropometric indicators (BMI, WHtR, WHR, BAI, CMI, LAP, VAI, AVI) were calculated, and statistical results illustrated that all anthropometric indicators were substantially associated with MAFLD. The connection powerfully emerged and persisted with MAFLD independent of potential confounding factors after logistic analysis. WHtR exhibited the persistently largest OR by stepwise binary logistic regression analysis regardless of potential confounders, suggesting WHtR may be defined as a critical anthropometric indicator that had optimal advantages in the prediction of MAFLD over the remainders. In addition to ROC curve analysis, although LAP had the largest AUC [0.868(95\% Cl 0.853-0.883)], it intimately followed by WHtR [0.863(95\% Cl 0.848-0.879)], the latter can be regarded as consubstantially to have the best diagnostic value of MAFLD. The rationale to grounded WHtR as the strongest diagnostic predictor for MAFLD was sufficient justified. The study showed additional surprises. AVI, a previously neglected indicator, in ORs and AUCs ranked second and third respectively, with OR 19.275(95\% Cl 14.266-26.041) and AUC $0.859(95 \% \mathrm{Cl}$ 0.843-0.874) identified, indicating excellent predictive and diagnostic power of MAFLD as well, deserves greater attention.

Heretofore, numerous studies had been demonstrated as anthropometric indicators having extent predictive capabilities for various fields of health conditions and chronic diseases such as central adiposity, diabetes mellitus, cardiometabolic diseases, left ventricular hypertrophy, and hyperuricemia [12]. A latest systematic review and dose-response meta-analysis stressed the indices of central fatness (WC, WHR, WHtR, BAI, ABSI and waist-to-thigh ratio) were positively and significantly associated with higher all-cause mortality risk [15]. Yet few studies adequately evaluate the diagnostic abilities of anthropometric indicators in predictive of MAFLD.

BMI used to be a surrogate index of visceral adiposity [41], whereas its validity as an appropriate indicator of obesity has been criticized for it cannot differentiate lean body mass and fat mass, fails to characterize regional fat distribution, racial/ethnic heterogeneity and varies by gender despite comparable body fat proportion [15, 24]. Nevertheless, BMI is still widely used in the evaluation of NAFLD individuals [11]. The prevalence of NAFLD is proportional to the increase in BMI [8]. Ju et al. investigated 9159 adults Koreans in a cross-sectional study with BMI of $22.38 \pm 2.53 \mathrm{~kg} / \mathrm{m}^{2}$ in Non-NAFLD group $(\mathrm{n}=6606)$ and $25.63 \pm 2.61 \mathrm{~kg} / \mathrm{m}^{2} \mathrm{in}$ NAFLD group $(n=2553)$, the AUC of BMI in NAFLD were 0.760 (95\% Cl 0.747-0.773) in men and $0.830(95 \% \mathrm{Cl} 0.811-0.850)$ in women [42]. Zheng et al. conducted a cross-sectional study in Xiamen with 490 Chinese and exhibited the results with BMI $24.78 \pm 10.14$ $\mathrm{kg} / \mathrm{m}^{2}$ in Non-NAFLD group $(\mathrm{n}=240)$ and $36.79 \pm 10.12 \mathrm{~kg} / \mathrm{m}^{2}$ in NAFLD group $(\mathrm{n}=250)$, in addition to the AUC and cut-off point of BMI in predicting NAFLD reached 0.854 (95\% $\mathrm{Cl} 0.78-0.93$ ) and $24.22 \mathrm{~kg} / \mathrm{m}^{2}$ (sens: $96 \%$, spec: $64 \%$ ) in total sample(containing male and female), respectively[43]. The findings of our study, BMI presented as $22.44 \pm 2.92 \mathrm{~kg} / \mathrm{m}^{2}$ in Non-MAFLD group $(n=1840)$ and $26.49 \pm$ $2.82 \mathrm{~kg} / \mathrm{m}^{2}$ in MAFLD group $(\mathrm{n}=515)$. The AUC and cut-off point of BMI in predicting MAFLD were $0.846(95 \% \mathrm{Cl} 0.829-0.864)$ and $24.0 \mathrm{~kg} / \mathrm{m}^{2}$ (sens: $84.08 \%$, spec: $72.39 \%$ ), separately. Although BMI remained that with reliable predictive value and satisfactory sensitivity and specificity for MAFLD, yet absented complete dominance compare to WHtR, as aforementioned in the present results.

Varying research findings existed regarding the prediction efficacy of WHR and WHtR for NAFLD, nonetheless, those results are too limited to be conclusive. Although the limited number of available studies were found that addressed the prediction efficacy and diagnostic competency of WHR and WHtR in NAFLD, the WHR had been determined the indicator with the highest diagnostic value [AUC:0.916(95\% Cl0.86-0.97)] for NAFLD in the study performed by Zheng et al.[43], with a cut-off point of 0.89 (sens: $99 \%$, spec: $66 \%)$ for the total sample, compare to WHtR[0.878 (95\% Cl 0.82-0.94);0.49(sens:96\%,spec:64\%)].Coincidentally,the findings of the mentioned above study about cut-off points and ours [WHR: 0.89(sens:84.46\%,spec:64.95\%);WHtR:0.49(sens:90.68\%,spec:69.56\%)] are overall consistent, despite the slightly lower sensitivity. Inconsistently, our study clarified that the indicator balancing both strongest prediction performance and optimal diagnostic capability for MAFLD is WHtR, as we mentioned above. There are diverse results had been reported. Motamed et al. used a cross-sectional study with 4872 Iranian adults, reported an AUC above 0.70 in WHR as well as above 0.80 in WHtR with both sexes to detect NAFLD, emphasized that the diagnostic power of WHtR was higher than WHR [12], which is consistent with our results. The optimal cut-off points for WHtR were 0.53 (sens: $82.7 \%$, spec: $70.8 \%$ ) for men and 0.58 (sens: $83.3 \%$, spec: 71.7\%) for women while for WHR were not presented [12]. Yoo et al. analyzed a observational cohort including 456 subjects, identified AUC of WHtR in NAFLD above 0.70 in total sample and the cut-off points presented 0.52 (sens:71\%, spec:65\%) for men and 0.53 (sens:90\%, spec: 63\%) for women [44]. Despite the inconsistent findings presented among the above studies may be partially attributed to the racial/ethnic discrepancy along with economic and geographic diversification, WHtR and WHR can be regarded as outstanding predictors for MAFLD undoubtedly. Notably, the findings of Zhang et al. [43] were conducted on a population with high proportion of males and our study was performed completely by a male survey, the cut-off points of WHR and WHtR we shared consistently may suggest more useful in the discrimination of MAFLD in males.

LAP expressed a continuous marker of lipid over-accumulation, which had been proposed to be a good predictor for cardiovascular disease and diabetes [26]. VAl expressed the visceral fat function associated with cardiometabolic risk and a predictor of 
complications related to visceral obesity [19]. Both LAP and VAI were defined as novel sex-specific indices which had high accuracy in visceral obesity discrimination and were effective in cardiovascular risk assessment, especially tended to elderly men [21]. In literature, up to now, only two studies have reported the prediction of NAFLD concerning LAP. Cuthbertson et al. demonstrated an AUC of 0.78 without calculated cut-off points for NAFLD by evaluated 4 cohorts from Germany and England [45]. A large cross-sectional study utilized 40459 Chinese in Changsha had already highlighted, which LAP showed high accuracy for discriminating NAFLD in both males and females (AUCs 0.843 and 0.887 , respectively), with cut-off points of 30.5 (sens:77\%, spec: $75 \%$ ) and 23.0 (sens:82\%, spec: $79 \%$, respectively [46]. Similarly, according to the present study, LAP had the largest AUC [0.868( $95 \% \mathrm{Cl} 0.853-0.883)]$ which indicating the highest diagnostic value of MAFLD compare to the remainders. As for the cut-off points substantially lower than Dai et al. [46] (24.29 vs.30.5 in males) may be due to regional economic gap and high disparity in area utilization. The diagnostic effectiveness of VAl in NAFLD individuals remains controversial. Vongsuvanh et al. [47], Díez-Rodríguez et al. [48] and Ercin et al. [49] claimed that VAI was no more powerful than WC in discriminating hepatic steatosis from steatohepatitis and not associated with liver histology either. While Patta et al. reported VAI was a marker of IR, both qualitative and quantitative of adipose tissue dysfunction, which significantly correlated with fibrosis in NAFLD patients [50]. Our present study demonstrated the AUC of VAl was $0.807(95 \% \mathrm{Cl} 0.787-0.826)$ in MAFLD subjects, yet VAl still ranked as posterior position in all indicators regardless of predictive capability or diagnostic value.

Of the remainders, $\mathrm{AVI}, \mathrm{CMI}$ and $\mathrm{BAI}$ have not been applied as predictors for NAFLD in the existing literature to date. AVI was suggested to estimated overall abdominal volume, which is strongly related to impaired glucose tolerance and diabetes [27]. Yet the study about the effectiveness of $\mathrm{AVI}$ has not been reported so far. CMI was proposed as a good predictor and discriminator of a central component of metabolic syndrome and diabetes, reflecting both adiposity and blood lipids [26]. Cantero et al. reported that the substantial decline of CMI could achieve the largest decrease in TG, LDL-C, total and visceral fat mass [51]. Verma et al. gave an insight that BAI was observed to be the most sensitive and specific indicator for predict overweight and obesity in females [0.902(95\% $\mathrm{Cl} 0.877-0.927)]$, while WHtR was the most reliable and sensitive surrogate indicator of obesity in males [AUC: $0.914(95 \% \mathrm{Cl} 0.891-0.936)$ ], compared to WC, BMI and WHR [14]. The ROC curve analysis in our study completely empowered AVI, CMI and BAI with new vision in discrimination of MAFLD. Superior diagnostic values of AVI, CMI and BAI were stressed as AUCs all above 0.79.The diagnostic capability of AVI and CMI (AUCs: 0.859 and 0.819) is even better than WHR. As an unexpected harvest, AVI should be warranted further study for its outstanding predictive and diagnostic performance in MAFLD as addressed above. Several new indices of adipose accumulation, including $\mathrm{ABSI}$ and $\mathrm{BRI}$ did not integrate into our analysis due to the limitation of their cumbersome formulas and proven poorly diagnostic power for NAFLD compare to WHtR and WHR [12].

Insulin resistance (IR) occurs as the cardinal feature of both excess adiposity and NAFLD [24]. The lipotoxicity produced by the fatty acids from dysfunctional and insulin-resistant adipocytes derived from the accumulation of triglyceride-derived toxic metabolites in ectopic tissues (skeletal muscle, pancreas, liver, etc.) [9]. Ultimately, IR triggers compensatory hyperinsulinemia, which intimately activated de novo hepatic lipogenesis, further exacerbating NAFLD [24]. Noteworthy, across racial/ethnic groups, IR presented differential manifestations. Report from the Dallas Heart Study showing a decreased prevalence of NAFLD among non-Hispanic black population despite high IR, which suggests that the intersection of IR and metabolic abnormalities on NAFLD has variable impacts among the different racial/ethnic groups [24]. Existing studies established that HOMA-IR, HOMA- $\beta$ and HOMA-IS were used to evaluate IR evaluation, insulin secretion and insulin sensitivity, respectively [52]. In our previous research, the extent of HOMA-IR and HOMA- $\beta$ was significantly correlated with the severity of NAFLD. The moderate-severe NAFLD group exhibited higher HOMA-IR (4.16 \pm 2.84 vs. $2.38 \pm 1.35, p<0.001)$ and HOMA- $\beta(162.04 \pm 139.29$ vs. $115.72 \pm 71.10, p=0.003)$ than mild NAFLD group [31]. The present analysis demonstrated that individuals with MAFLD had higher HOMA-IR [2.55(1.80-3.75) vs. $1.29(0.87-1.90), p<0.001]$ and HOMA- $\beta$ level [115.08(79.45-164.45) vs. 69.82(45.86-104.74), $p<0.001]$ while lower HOMA-IS value [0.39(0.27-0.56) vs. 0.78(0.53-1.14), $p<0.001]$ compared to those without MAFLD, which was mostly consistent with previous findings. When taking into account HOMA-IR and HOMA- $\beta$ as the indicators of predictive capacity for MAFLD, their AUCs are above 0.70 with cut-off points determined high sensitivity and poor specificity.

Ideally, liver histological (biopsy) remains the gold standard for diagnosis of NAFLD, but its various defects like invasive nature,expenditure, sampling errors and potential complications, clearly indicating the impractical for routinely screen[2,5]. Consequently, non-invasive detection mothed for NAFLD relies on blood biomarkers and imaging techniques. Despite its limited sensitivity, ultrasonography contains the most widely used methodology as the first-line diagnostic modality for NAFLD. Computed tomography and magnetic resonance imaging are available and more precisely, yet still not widely used in large-scale populations or clinical settings for the reasons including unequivocally higher cost and the ionizing radiation. Blood biomarkers appear only to be appropriate for extensive epidemiological studies, remain inconvenient for public healthy prediction and evaluation [1]. Comparably,

Page 12/20 
our study contributing that the clinical anthropometric indicators of visceral obesity, especially WHtR, of easy applicability and costeffective, have been very promising in the prediction and discrimination of MAFLD, particularly in the less economically developed districts of China.

\section{Study strengths and limitations}

This is the first large cross-sectional study that uses a Chinese male population to rigorously highlight and compare the predictive capability of eight anthropometric indicators of visceral adiposity in MAFLD individuals. And we revealed that all selected anthropometric indicators do bolster optimism in valuable diagnostic capacity for MAFLD. Furthermore, we located the optimal diagnostic predictor and its successors for MAFLD.

The study has several limitations that should be acknowledged. Since the starting point of FAMHES was focusing on male health and investigating the effects of genetic and environmental factors of age-related chronic diseases in men, we lacked female subjects enrolled in this study and absented gender comparisons. Secondly, the study was a retrospective cross-sectional design and could not draw out the identification of causal relationships. Thirdly, the limited available literature evaluated the association between anthropometric indicators and liver fat deposition were fully conducted with NAFLD populations as the original reference group. Correspondingly, the discrepancy of the population between NAFLD and MAFLD might lead to some extent bias and confusion when citing relevant results.

\section{Conclusion}

To summarize, our study detailed the significant impact of MAFLD prevalence in Western China and simultaneously underscored the effect of race/ethnic heterogeneity and regional variations in the anthropometric indicators and their associations with MAFLD. All eight anthropometric indicators illustrated the superior diagnostic value of MAFLD with AUCs fully above 0.79. Principally, we stressed WHtR as the most powerful diagnostic predictor for MAFLD, while LAP and AVI presented highly potential only after WHtR. The present findings representing promising applied tools with widespread availability and high reproducibility may beneficial for the early prediction and discrimination of MAFLD, especially true for underdeveloped, resource scarce regions.

\section{Abbreviations}

NAFLD: Non-alcoholic fatty liver disease; MAFLD: Metabolic-dysfunction associated fatty liver disease; FAMHES: Fangchenggang Area Male Health and Examination Survey; BMl: Body mass index; WHtR: Waist-to-height ratio; WHR: Waist-hip ratio;

BAI: Body adiposity index; CMI: Cardiometabolic index; LAP: Lipid accumulation product; VAl: Visceral adiposity index; AVI: Abdominal volume index; ROC: Receiver operating characteristic curve; AUC: Area under the ROC curves; ORs: Odds ratios; Cl: Confidence interval; WC: Waist circumference; BRI: Body roundness index; ABSI: Body shape index; HC: Hip circumference; SBP: Systolic blood pressure; DBP: Diastolic blood pressure; TG: Triglyceride; TC: Total cholesterol; HDL: High-density lipoprotein; LDL: Low-density lipoprotein; ALT: Alanine aminotransferase; UA: Uric acid; FPG: Fasting plasma glucose; FSH: Follicle stimulating hormone; LH: Luteinizing hormone; TT: Total testosterone; E2: Estradiol; SHBG: Serum sex hormone-binding globulin; IR: Insulin resistance; HOMA: Homeostatic model assessment; HOMA-IS: HOMA\% Sensitivity ; GDP: Gross domestic product. sens: sensitivity; spec: specificity

\section{Declarations}

\section{Acknowledgements}

This research was conducted using database from Guangxi Medical University Genomic and Personalized Medicine Center of Biological Sample Biobank and Bioinformatics Database Sharing Service Platform.

\section{Authors' contributions}

JWC and CTL conceptualized and designed the study, harvested the major statistical analysis, drafted the initial manuscript. SQL and YSL assisted in statistical analysis, data interpretation and demonstration. ML, YFQ and XHL assisted in reviewed and revised the manuscript. AHT, YG, ZL, CLW, SZH, XBY and HYZ gathered and marshalled the data. ZNM and JK conceptualized and administered 
the study, mentoring supervision of the study and data analysis, critically reviewed and revised the initial manuscript. All authors consented the final manuscript submitted.

\section{Funding}

This study was supported by grants from Guangxi key Laboratory for Genomic and Personalized Medicine (grant number 16-38054, 17-259-45, 19-050-22, 19-185-33, 20-065-33); National Key Research \& Development Projects (2018YFC1314104) and Guangxi Natural Science Fund (2017GXNSFAA198097).

\section{Availability of data and materials}

All data used in this study are available from the corresponding author.

\section{Declarations}

\section{Ethics approval and consent to participate}

The study received approval by the Medical Ethics Committee of First Affiliated Hospital of Guangxi Medical University. (No. 2008(KY004))

\section{Consent for publication}

Not applicable.

\section{Competing interests}

The authors have declared no potential conflicts of interest exist.

\section{Author details}

${ }^{1}$ The Second School of Clinical Medicine, Southern Medical University, Guangzhou, Guangdong, China

2 Department of Endocrinology, Guangdong Provincial People's Hospital, Guangdong Academy of Medical Sciences, Guangzhou, Guangdong, China

${ }^{3}$ Department of Endocrinology, The First Affiliated Hospital of Guangxi Medical University, Nanning, Guangxi Zhuang Autonomous Region, China

${ }^{4}$ School of Pharmaceutical Sciences, Southern Medical University, Guangzhou, Guangdong, China

${ }^{5}$ Department of Neurology, First Affiliated Hospital of Guangxi Medical University, Nanning, Guangxi Zhuang Autonomous Region, China

${ }^{6}$ Center for Genomic and Personalized Medicine, Guangxi Medical University, Nanning, Guangxi Zhuang Autonomous Region, China

${ }^{7}$ Guangxi Key Laboratory of Genomic and Personalized Medicine, Nanning, Guangxi Zhuang Autonomous Region, China

${ }^{8}$ Guangxi Collaborative Innovation Center for Genomic and Personalized Medicine, Nanning, Guangxi Zhuang Autonomous Region, China

${ }^{9}$ Institute of Urology and Nephrology, First Affiliated Hospital of Guangxi Medical University, Nanning, Guangxi Zhuang Autonomous Region, China

\section{References}

1. Eslam M, Newsome PN, Sarin SK, Anstee QM, Targher G, Romero-Gomez M, et al.A new definition for metabolic dysfunctionassociated fatty liver disease: An international expert consensus statement. J Hepatol 2020, 73:202-209. 
https://doi.org/10.1016/j.jhep.2020.03.039.

2. Lee SJ, Kim SU.Noninvasive monitoring of hepatic steatosis: controlled attenuation parameter and magnetic resonance imagingproton density fat fraction in patients with nonalcoholic fatty liver disease. Expert Rev Gastroenterol Hepatol 2019, 13:523530.https://doi.org/10.1080/17474124.

2019.1608820.

3. Eslam M, Sanyal AJ, George J, on behalf of an International Consensus Panel.MAFLD: A Consensus-Driven Proposed Nomenclature for Metabolic Associated Fatty Liver Disease. Gastroenterology 2020, 158:1999-2014 e1991.

https://doi.org/10.1053/j.gastro.2019.11.312.

4. Li J, Zou B, Yeo YH, Feng Y, Xie X, Lee DH, et al. Prevalence, incidence, and outcome of non-alcoholic fatty liver disease in Asia, 1999-2019: a systematic review and meta-analysis. The Lancet Gastroenterology \& Hepatology 2019, 4:389398.https://doi.org/10.1016/s2468-1253(19)30039-1.

5. Zhou J, Zhou F, Wang W, Zhang XJ, Ji YX, Zhang P, et al. Epidemiological Features of NAFLD From 1999 to 2018 in China. Hepatology 2020, 71:1851-1864. https://doi.org/10.1002/hep.31150.

6. Sun DQ, Jin Y, Wang TY, Zheng KI, Rios RS, Zhang HY, et al.MAFLD and risk of CKD. Metabolism 2021, 115:154433.https://doi.org/10.1016/j.metabol.2020.

154433.

7. Lin S, Huang J, Wang M, Kumar R, Liu Y, Liu S,et al.Comparison of MAFLD and NAFLD diagnostic criteria in real world. Liver Int 2020, 40:2082-2089. https://doi.org/10.1111/liv.14548.

8. Younossi ZM.Non-alcoholic fatty liver disease - A global public health perspective. $J$ Hepatol 2019, 70:531544.https://doi.org/10.1016/j.jhep.2018.

10.033.

9. Engin A.Non-Alcoholic Fatty Liver Disease. Adv Exp Med Bio/ 2017, 960:443-467. https://doi.org/10.1007/978-3-319-483825_19.

10. Gustafson B, Smith U.Regulation of white adipogenesis and its relation to ectopic fat accumulation and cardiovascular risk. Atherosclerosis 2015, 241:27-35. https://doi.org/10.1016/j.atherosclerosis.2015.04.812.

11. Almeida NS, Rocha R, Cotrim HP, Daltro C.Anthropometric indicators of visceral adiposity as predictors of non-alcoholic fatty liver disease: A review. World J Hepatol 2018, 10:695-701. https://doi.org/10.4254/wjh.v10.110.695.

12. Motamed N, Rabiee B, Hemasi GR, Ajdarkosh H, Khonsari MR, Maadi M, et al.Body Roundness Index and Waist-to-Height Ratio are Strongly Associated With Non-Alcoholic Fatty Liver Disease: A Population-Based Study. Hepat Mon 2016, 16:e39575.https://doi.org/10.5812/hepatmon.39575.

13. Rotter I, Ryl A, Grzesiak K, Szylinska A, Pawlukowska W, Lubkowska A, et al.Cross-Sectional Inverse Associations of Obesity and Fat Accumulation Indicators with Testosterone in Non-Diabetic Aging Men. Int J Environ Res Public Health 2018, 15.https://doi.org/10.3390/ijerph15061207.

14. Verma M, Rajput M, Sahoo SS, Kaur N, Rohilla R.Correlation between the percentage of body fat and surrogate indices of obesity among adult population in rural block of Haryana. J Family Med Prim Care 2016, 5:154-159.

https://doi.org/10.4103/2249-4863.184642.

15. Jayedi A, Soltani S, Zargar MS, Khan TA, Shab-Bidar S.Central fatness and risk of all cause mortality: systematic review and dose-response meta-analysis of 72 prospective cohort studies. BMJ 2020, 370:m3324.https://doi.org/10.1136/

Page $15 / 20$ 
bmj.m3324.

16. WHO expert consultation. Appropriate body-mass index for Asian populations and its implications for policy and intervention strategies. The Lancet 2004, 363:157-163. https://doi.org/10.1016/s0140-6736(03)15268-3.

17. Kahn HS.The "lipid accumulation product" performs better than the body mass index for recognizing cardiovascular risk: a population-based comparison. BMC Cardiovasc Disord 2005, 5:26. https://doi.org/10.1186/1471-2261-5-26.

18. Peters SAE, Carcel C, Millett ERC, Woodward M.Sex differences in the association between major risk factors and the risk of stroke in the UK Biobank cohort study. Neurology 2020, 95:e2715-e2726.https://doi.org/10.1212/WNL.

\section{2.}

19. Amato MC, Giordano C, Galia M, Criscimanna A, Vitabile S, Midiri M,et al.Visceral Adiposity Index: a reliable indicator of visceral fat function associated with cardiometabolic risk. Diabetes Care 2010, 33:920-922. https://doi.org/10.2337/dc09-1825.

20. Bergman RN, Stefanovski D, Buchanan TA, Sumner AE, Reynolds JC, Sebring NG,et al.A better index of body adiposity. Obesity (Silver Spring) 2011, 19:1083-1089. https://doi.org/10.1038/oby.2011.38.

21. Roriz AK, Passos LC, de Oliveira CC, Eickemberg M, Moreira Pde A, Sampaio LR. Evaluation of the accuracy of anthropometric clinical indicators of visceral fat in adults and elderly. PLoS One 2014, 9:e103499.https://doi.org/10.1371/jour

nal.pone.0103499.

22. Thomas DM, Bredlau C, Bosy-Westphal A, Mueller M, Shen W, Gallagher D, et al.Relationships between body roundness with body fat and visceral adipose tissue emerging from a new geometrical model. Obesity (Silver Spring) 2013, 21:22642271.https://doi.org/10.1002/oby.20408.

23. Krakauer NY, Krakauer JC.A new body shape index predicts mortality hazard independently of body mass index. PLoS One 2012, 7:e39504.https://doi.org/

10.1371/journal.pone.0039504.

24. Agbim U, Carr RM, Pickett-Blakely O, Dagogo-Jack S.Ethnic Disparities in Adiposity: Focus on Non-alcoholic Fatty Liver Disease, Visceral, and Generalized Obesity. Curr Obes Rep 2019, 8:243-254.https://doi.org/10.1007/s13679-019-00349-x.

25. Li L, Liu DW, Yan HY, Wang ZY, Zhao SH, Wang B.Obesity is an independent risk factor for non-alcoholic fatty liver disease: evidence from a meta-analysis of 21 cohort studies. Obes Rev 2016, 17:510-519.https://doi.org/10.1111/obr.12407.

26. Wakabayashi I, Daimon T.The "cardiometabolic index" as a new marker determined by adiposity and blood lipids for discrimination of diabetes mellitus. Clin Chim Acta 2015, 438:274-278.https://doi.org/10.1016/j.cca.2014.08.042.

27. Guerrero-Romero F, Rodríguez-Morán M.Abdominal volume index. an anthropometry-based index for estimation of obesity is strongly related to impaired glucose tolerance and type 2 diabetes mellitus. Archives of Medical Research 2003, 34:428-432. https://doi.org/10.1016/s0188-4409(03)00073-0.

28. Tan A, Gao Y, Yang X, Zhang H, Qin X, Mo L, et al.Low serum osteocalcin level is a potential marker for metabolic syndrome: results from a Chinese male population survey. Metabolism 2011, 60:1186-1192.https://doi.org/10.1016/

j.metabol.2011.01.002.

29. Gao Y, Wang M, Zhang H, Tan A, Yang X, Qin X, et al.Are metabolic syndrome and its components associated with lower urinary tract symptoms? Results from a Chinese male population survey. Urology 2012, 79:194-201. https://doi.org/10.1016/j.urology.2011.07.1399.

30. Liu Z, Ye F, Zhang H, Gao Y, Tan A, Zhang S, et al. The association between the levels of serum ferritin and sex hormones in a large scale of Chinese male population. PLoS One 2013, 8:e75908.https://doi.org/10.1371/journal.pone. 
0075908.

31. Ye J, Yao Z, Tan A, Gao Y, Chen Y, Lin X, et al. Low Serum Sex Hormone-Binding Globulin Associated with Insulin Resistance in Men with Nonalcoholic Fatty Liver Disease. Horm Metab Res 2017, 49:359-364.https://doi.org/10.1055/s-0043-102690.

32. Tekin T, Cicek B, Konyaligil N, Gunturk I, Yazici C, Karaca Z, et al. Increased hip circumference in individuals with metabolic syndrome affects serum nesfatin-1 levels. Postgrad Med J 2020, 96:600-605.https://doi.org/10.1136/postgradme

dj-2019-136887.

33. Nishida C, Ko GT, Kumanyika S.Body fat distribution and noncommunicable diseases in populations: overview of the 2008 WHO Expert Consultation on Waist Circumference and Waist-Hip Ratio. Eur J Clin Nutr 2010, 64:2-5.https://doi.org/10.1038/ejcn.2009.139.

34. Tian GX, Sun Y, Pang CJ, Tan AH, Gao Y, Zhang HY,et al.Oestradiol is a protective factor for non-alcoholic fatty liver disease in healthy men. Obesity Reviews 2012, 13:381-387.https://doi.org/10.1111/j.1467-789X.2011.00978.x.

35. Habib SS, Alkahtani S, Alhussain M, Aljuhani O.Sarcopenia Coexisting with High Adiposity Exacerbates Insulin Resistance and Dyslipidemia in Saudi Adult Men. Diabetes Metab Syndr Obes 2020, 13:3089-3097.https://doi.org/10.2147/

DMSO.S260382.

36. Jung K, Kim J, Park HY, Jung WS, Lim K.Hypoxic Pilates Intervention for Obesity: A Randomized Controlled Trial. Int J Environ Res Public Health 2020, 17.

https://doi.org/10.3390/ijerph17197186.

37. Xie Y, Wang M, Zhang Y, Zhang S, Tan A, Gao Y, et al.Serum uric acid and non-alcoholic fatty liver disease in non-diabetic Chinese men. PLoS One 2013, 8:e67152.https://doi.org/10.1371/journal.pone.0067152.

38. Shan G.Improved Confidence Intervals for the Youden Index. PLoS One 2015, 10:e0127272.

https://doi.org/10.1371/journal.pone.0127272.

39. Seo IH, Lee HB, Kim S, Lee YJ, Jung DH.Inverse Relationship between Hepatic Steatosis and Alanine Aminotransferase with Sex Hormone-Binding Globulin in Men. Yonsei Med J 2017, 58:731-736.https://doi.org/10.3349/ymj.2017.58.4.

731.

40. Wang X, Xie J, Pang J, Zhang H, Chen X, Lin J, et al. Serum SHBG Is Associated With the Development and Regression of Nonalcoholic Fatty Liver Disease: A Prospective Study. J Clin Endocrinol Metab 2020, 105.https://doi.org/10.1210/

clinem/dgz244.

41. Bedogni G, Bellentani S, Miglioli L, Masutti F, Passalacqua M, Castiglione A,et al.The Fatty Liver Index: a simple and accurate predictor of hepatic steatosis in the general population. BMC Gastroentero/ 2006, 6:33.https://doi.org/10.1186

/1471-230X-6-33.

42. Ju DY, Choe YG, Cho YK, Shin DS, Yoo SH, Yim SH, et al.The influence of waist circumference on insulin resistance and nonalcoholic fatty liver disease in apparently healthy Korean adults. Clin Mol Hepatol 2013, 19:140-147. https://doi.org/10.3350/cmh.2013.19.2.140.

43. Zheng RD, Chen ZR, Chen JN, Lu YH, Chen J.Role of Body Mass Index, Waist-to-Height and Waist-to-Hip Ratio in Prediction of Nonalcoholic Fatty Liver Disease. Gastroenterol Res Pract 2012, 2012:362147.https://doi.org/10.1155/2012/

362147.

44. Yoo HJ, Park MS, Lee CH, Yang SJ, Kim TN, Lim Kl, et al.Cutoff points of abdominal obesity indices in screening for nonalcoholic fatty liver disease in Asians. Liver Int 2010, 30:1189-1196.https://doi.org/10.1111/j.1478-3231.

Page $17 / 20$ 
45. Cuthbertson DJ, Weickert MO, Lythgoe D, Sprung VS, Dobson R, Shoajee-Moradie F, et al.External validation of the fatty liver index and lipid accumulation product indices, using $1 \mathrm{H}$-magnetic resonance spectroscopy, to identify hepatic steatosis in healthy controls and obese, insulin-resistant individuals. Eur J Endocrinol 2014, 171:561-569. https://doi.org/10.1530/EJE-14-0112.

46. Dai H, Wang W, Chen R, Chen Z, Lu Y, Yuan H.Lipid accumulation product is a powerful tool to predict non-alcoholic fatty liver disease in Chinese adults. Nutr Metab (Lond) 2017, 14:49. https://doi.org/10.1186/s12986-017-0206-2.

47. Vongsuvanh R, George J, McLeod D, van der Poorten D.Visceral adiposity index is not a predictor of liver histology in patients with non-alcoholic fatty liver disease. J Hepatol 2012, 57:392-398.https://doi.org/10.1016/j.jhep.2012.03.

013.

48. Rubén DR, María DB, Alicia CF,Tomás GDF , Luis GH, Sara CA,et al.Insulin resistance and metabolic syndrome are related to nonalcoholic fatty liver disease, but not visceral adiposity index, in severely obese patients.Rev Esp Enferm Dig 2014, 106:522528.https://doi.org/PMID:25544409.

49. Ercin CN, Dogru T, Genc H, Celebi G, Aslan F, Gurel H, et al.Insulin Resistance but Not Visceral Adiposity Index Is Associated with Liver Fibrosis in Nondiabetic Subjects with Nonalcoholic Fatty Liver Disease. Metab Syndr Relat Disord 2015, 13:319325.https://doi.org/10.1089/met.2015.0018.

50. Petta S, Amato MC, Di Marco VD, Camma C, Pizzolanti G, Barcellona MR,et al.Visceral adiposity index is associated with significant fibrosis in patients with non-alcoholic fatty liver disease. Aliment Pharmacol Ther 2012, 35:238-247.

https://doi.org/10.1111/j.1365-2036.2011.04929.x.

51. Kendel Jovanovic G, Mrakovcic-Sutic I, Pavicic Zezelj S, Susa B, Rahelic D, Klobucar Majanovic S.The Efficacy of an EnergyRestricted Anti-Inflammatory Diet for the Management of Obesity in Younger Adults. Nutrients 2020, 12.

https://doi.org/10.3390/nu12113583.

52. Yang S, Zhao H, Xu K, Qian Y, Wu M, Yang T, et al. Evaluation of common variants in MG53 and the risk of type 2 diabetes and insulin resistance in Han Chinese. Springerplus 2016, 5:612.https://doi.org/10.1186/s40064-016-2218-1.

\section{Figures}




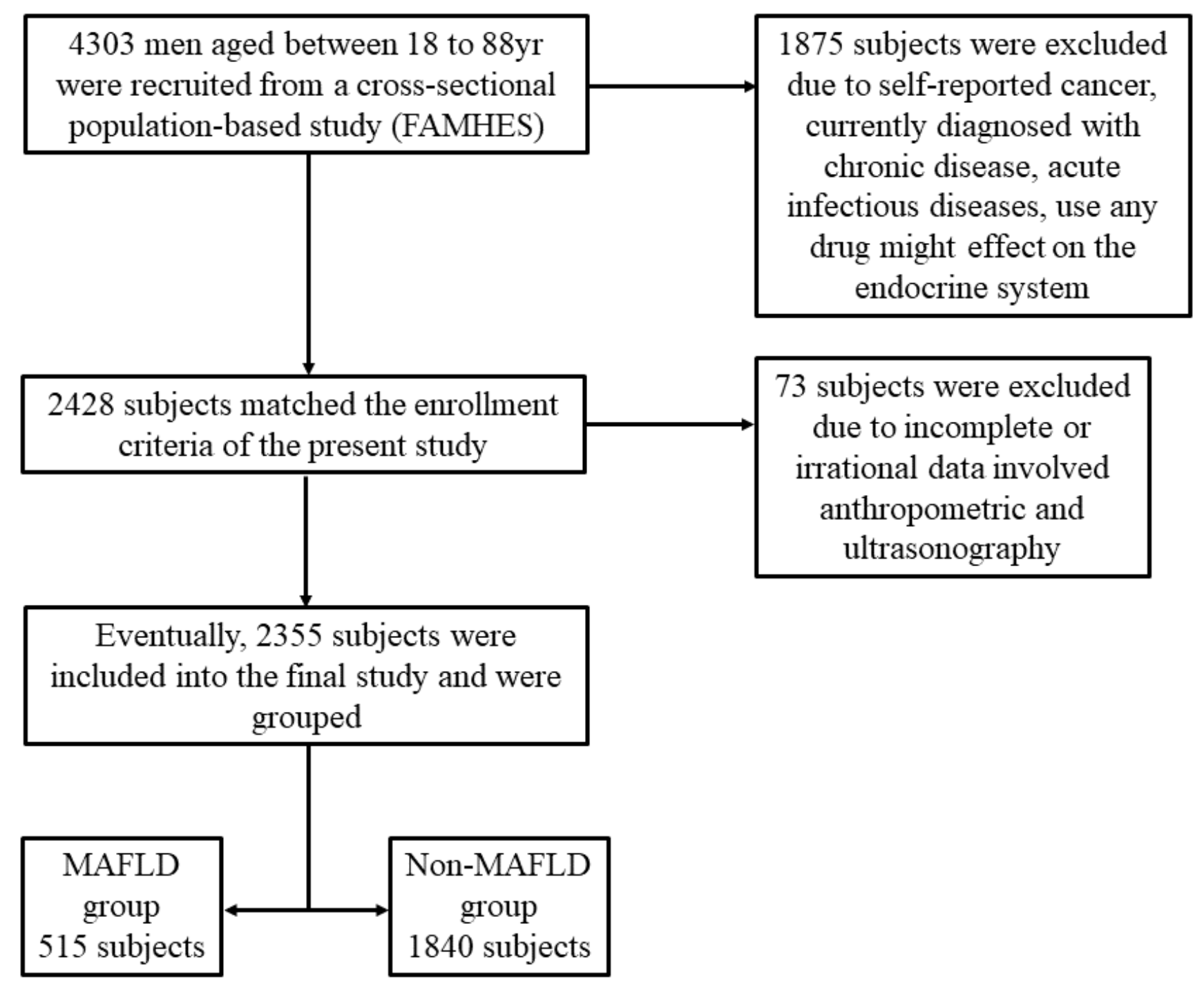

Figure 1

Flowchart of the study participants and exclusions. 


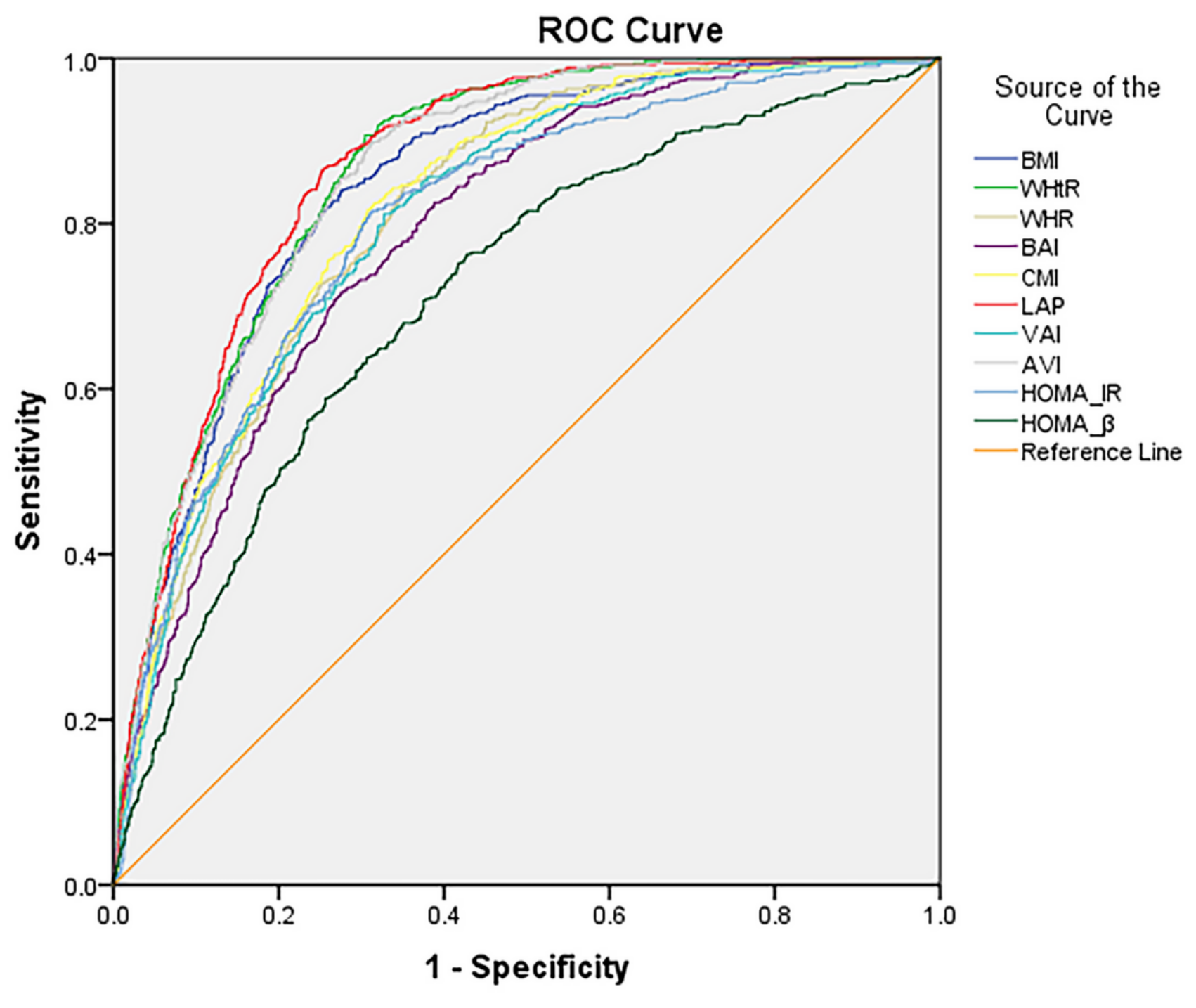

Diagonal segments are produced by ties.

Figure 2

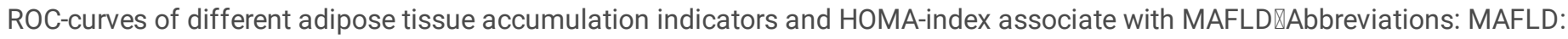
Metabolic-dysfunction associated fatty liver disease; BMI: Body mass index; WHtR: Waist to height ratio; WHR: Waist to hip ratio; BAI: Body adiposity index; CMI: Cardiometabolic index; LAP: Lipid accumulation product; VAl: Visceral adiposity index; AVI: Abdominal volume index; HOMA: Homeostatic model assessment 